\title{
Um Método para Geração de Modelo de Sistemas-de-Sistemas de Informação a partir da Análise de Modelos de Processos de Negócio
}

\author{
Lucas da Silva Oliveira $^{1}$ (Mestrando), Aline Pires Vieira de Vasconcelos ${ }^{1}$ \\ (Orientadora), Rodrigo Pereira dos Santos $^{2}$ (Coorientador)
}
${ }^{1}$ Mestrado Profissional em Sistemas Aplicados à Engenharia e Gestão (SAEG)
Programa de Pós-Graduação em Sistemas Aplicados à Engenharia e Gestão
Instituto Federal de Educação, Ciência e Tecnologia Fluminense (IFF)
Rua Dr. Siqueira, 273 - CEP 28030-130 - Campos dos Goytacazes - RJ - Brasil
${ }^{2}$ Programa de Pós-Graduação em Informática (PPGI)
Universidade Federal do Estado do Rio de Janeiro (UNIRIO)
Avenida Pasteur, 458 - CEP 22290-250 - Rio de Janeiro - RJ - Brasil
Ingresso: 03/2019 - Qualificação:10/2020 - Previsão Defesa: 02/2021
oliveira.l.s.ec@gmail.com, apires@iff.edu.br, rps@uniriotec.br

\begin{abstract}
Resumo. As organizações buscam atingir metas pela execução de processos de negócio apoiados pelos sistemas de informação. Porém, muitas vezes os profissionais têm dificuldade em executar alguns processos de negócio pela falta de comunicação efetiva entre os sistemas, o que afeta as tarefas dos stakeholders. Recentemente, este arranjo de sistemas tem sido investigado como Sistema-de-Sistemas de Informação (SoIS), uma classe de Sistemas-deSistemas (SoS) focada em negócios. Entender processos de negócio se torna crítico para assegurar a interoperabilidade entre os sistemas. Esta pesquisa tem como objetivo desenvolver uma abordagem baseada em SoIS para detectar necessidades de interoperabilidade entre os sistemas de uma organização, ou de diferentes organizações, a partir da análise de processos de negócio de suas unidades. Foi realizado um levantamento bibliográfico por trabalhos nos temas de SoIS, interoperabilidade e BPMN onde foram encontrados dois mapeamentos sistemáticos recentes no assunto. A partir dos conhecimentos adquiridos com os trabalhos levantados e com estudos de diferentes exemplos de processos modelados em BPMN, foram desenvolvidas versões iniciais do método proposto neste trabalho e do ferramental de apoio a sua execução, assim como hipóteses de identificação de links de interoperabilidade entre sistemas. Um estudo de viabilidade foi realizado para avaliar estas hipóteses e atualizações no método e ferramenta estão sendo desenvolvidas baseadas nos resultados deste estudo e em conceitos de rastreabilidade e arquitetura de SoIS. Sendo assim, essa pesquisa apresenta a proposta de um método para geração de uma representação arquitetural de um SoIS a partir da análise de processos de negócio modelados em BPMN.
\end{abstract}

Palavras-chave. Sistema-de-Sistemas de Informação; Processo de Negócio; BPMN.

Evento CBSoft: $S B E S$. 


\section{Caracterização do Problema}

Organizações dos setores público e privado procuram gerar valor em seus produtos e serviços. Para isso, elas estabelecem metas e objetivos a serem cumpridos a partir da realização de seus processos de negócio, que têm a sua execução apoiada pelos seus sistemas de informação (SI). A fim de atingir os seus objetivos e missões, as organizações precisam lidar com diferentes aspectos técnicos, sociais e de negócio que, muitas vezes, afetam e são afetados por problemas de integração dos sistemas.

O isolamento dos sistemas impede a automação da entrega e sincronização de informações, reduz a associação e compartilhamento de dados entre sistemas e obstrui a comunicação entre diferentes setores, fazendo com que a organização acabe oferecendo aos clientes informações inconsistentes [FU et al. 2010]. Além disso, a falta de integração entre os sistemas aumenta os custos operacionais, de gerenciamento e de coordenação [GEORGANTZAS e KATSAMAKAS 2010]. Nesse sentido, para apoiar efetivamente os processos de negócio das organizações, os sistemas existentes devem ser integrados e interoperar, formando arranjos de SI [HASSELBRING 2000].

Mais recentemente, esse arranjo tem sido chamado de Sistema-de-Sistemas de Informação (do inglês, System-of-Information Systems, SoIS), que resulta de vários SI, intensivos em software ou não, operacionalmente e gerencialmente independentes que trabalham juntos para atingir objetivos de negócio comuns [MAJD et al. 2015]. Para tal, as relações entre estes SI interoperáveis são de extrema importância, uma vez que as empresas podem ser sustentadas e gerar mais valor se essas relações são melhor investigadas e compreendidas [SANTOS et al. 2018]. Como exemplo de SoIS, tem-se os diferentes sistemas de uma instituição de ensino que, apesar de serem independentes entre si, trabalham em conjunto para alcançar as suas diferentes metas organizacionais.

Uma vez que SoIS é uma classe de Sistemas-de-Sistemas (SoS), problemas de integração emergem ao lidar com a complexidade das interações entre os seus componentes. A identificação de processos de negócio que capturam as interações, procedimentos e requisitos que o sistema precisa atender se torna chave para $\mathrm{o}$ desenvolvimento bem-sucedido da arquitetura [KRUEGER et al. 2006]. Pelo fato dos SoIS apresentarem uma natureza predominantemente orientada a negócios, são necessárias metodologias específicas para capturar as características, sendo modelagem de processos de negócio potencialmente útil [GRACIANO NETO et al. 2017].

\section{Fundamentação Teórica}

Segundo Maier (1998), SoS são compostos por sistemas que apresentam independência operacional e gerencial e constituem uma classe de sistemas de larga escala e complexos que se originam da interoperabilidade de vários sistemas, chamados sistemas constituintes [LANA et al. 2016]. Por sua vez, SoIS representam um tipo específico de SoS composto por um ou mais SI intensivos em software ou não [TEIXEIRA et al. 2019]. SoIS representam uma classe de SoS compostos por SI pré-existentes. Ou seja, constitui-se em uma rede dinâmica de SI independentes que concretiza uma junção intra e inter-organizacional para prover serviços que não conseguiriam ser disponibilizados por nenhum dos sistemas de forma independente [FERNANDES et al. 2019].

SoIS é fruto dos processos de negócio que se modernizam e de um mundo globalizado onde diferente SI são forçados a interoperar com outros e visa disponibilizar serviços, firmar parcerias comerciais com fornecedores, cumprir as leis e entregar 
produtos de forma ágil e otimizada [TEIXEIRA et al. 2019]. Uma vez que os SoIS são orientados a processos de negócio, eles atendem a requisitos de negócio (alinhados a um ou mais processos) mapeados a um objetivo principal e a objetivos secundários relacionados ao SoIS e seus constituintes [FERNANDES et al. 2019].

Neste contexto, a modelagem de processos de negócio busca dar suporte a processos de negócio por meio de métodos, técnicas e software para projetar, executar, controlar e analisar processos operacionais, envolvendo humanos, organizações, aplicativos, documentos e outras fontes de informação [VAISMAN 2013]. Nesse contexto, BPMN (Business Process Modeling and Notation), padrão criado pelo Object Management Group (OMG), tem sido incluído entre as principais ferramentas de modelagem por apresentar um conjunto robusto de símbolos para modelar diferentes aspectos de processos de negócio [ABPM 2013].

BPMN é uma notação simples e expressiva, baseada em uma abordagem orientada a processos e amplamente utilizada na academia e na indústria para a representação do conhecimento sobre processos de negócio [ABPM 2013]. Esta notação apresenta elementos gráficos padronizados com base em técnicas de fluxograma [FALCONE et al. 2018], sendo de fácil entendimento, além de ser melhor apoiada em termos de ferramentas de execução [MENDES et al. 2018].

\section{Objetivo e Contribuições}

Esta pesquisa tem como objetivo geral desenvolver uma abordagem baseada em SoIS para detectar necessidades de interoperabilidade entre os SI de uma organização, ou de diferentes organizações, a partir da análise de processos de negócio de várias unidades organizacionais. A meta é ajudar gestores a identificarem problemas em cumprir metas com base na representação arquitetural do SoIS gerado. Pretende-se gerar as seguintes contribuições: (1) permitir a compreensão dos processos de negócio da organização e dos SI que os apoiam; (2) propor um método que permita elaborar uma representação arquitetural dos SI da organização na perspectiva de um SoIS; e (3) desenvolver um ferramental de apoio ao método proposta na dissertação.

\section{Metodologia}

Esta pesquisa parte das seguintes questões: "Como modelos de processos de negócio podem refletir pontos de interoperação entre os sistemas de uma organização?" e "Como gerar a representação arquitetural do arranjo de sistemas resultante com base nesses pontos de interoperação?". A pesquisa foi estruturada em duas fases principais.

A fase de concepção compreende as etapas necessárias para o desenvolvimento do método que visa à elaboração de uma representação arquitetural de SoIS, sendo estas: (1) Levantamento bibliográfico, onde foram feitas pesquisas nas bases de dados Scopus, ACM DL, IEEE Xplore e Web of Science pelos temas SoIS, Interoperabilidade e BPMN. Foram encontrados dois mapeamentos sistemáticos recentes no assunto [FERNANDES et al. 2019; TEIXEIRA et al. 2019], de modo que não se julgou ser necessário um novo estudo secundário neste mestrado; (2) Estudo de diferentes exemplos de processos modelados em BPMN com a finalidade de aprender a identificar pontos de interação entre diferentes unidades organizacionais, ou entre diferentes organizações, e os seus sistemas (i.e., links de interoperabilidade [FERNANDES et al. 2019]), a partir das tarefas realizadas por cada ator nos processos; (3) Desenvolvimento 
do método e ferramental de apoio iniciais para extração de dados de modelos de processos de negócio e identificação dos links de interoperabilidade entre os sistemas; (4) Realização em um estudo de viabilidade com o intuito de avaliar hipóteses de identificação de links de interoperabilidade, desenvolvidas com base nos conhecimentos adquiridos e aplicadas na análise de modelos de processos de negócio de um setor de uma instituição de ensino superior, com base em seus resultados está sendo planejado um estudo de maior amplitude envolvendo os usuários dos sistemas de diversos setores; (5) Avaliação dos resultados do estudo de viabilidade, em que se obteve indícios da viabilidade das hipóteses desenvolvidas assim como pontos de aperfeiçoamento para a versão inicial do método e ferramental de apoio; (6) Estudo de conceitos de rastreabilidade e arquitetura de SoIS que serão utilizados na geração de uma representação arquitetural do arranjo dos sistemas de uma organização, apresentando rastreabilidade entre elementos da arquitetura e os processos de negócio; e (7) Evolução do método e ferramental de apoio a partir dos resultados do estudo de viabilidade e dos conceitos de rastreabilidade e arquitetura de SoIS. As etapas de 1 a 5 estão concluídas e as etapas 6 e 7 se encontram em execução no momento.

Em relação à fase de avaliação, pretende-se verificar a efetividade do método proposto com base em um estudo de caso em uma organização que apresente as seguintes características: (i) comunicação e colaboração entre diferentes unidades organizacionais, ou entre diferentes organizações, durante a execução de processos de negócio; e (ii) uso de mais de um SI para apoiar a execução destes processos. A organização selecionada é um instituto federal de educação cujos processos de negócio dependem da interação entre diferentes unidades e outras organizações externas. A representação arquitetural da organização gerada e os demais resultados obtidos com o uso do método serão verificados por especialistas da área de SoS/SoIS. Por sua vez, será aplicado um questionário com diferentes stakeholders da organização visando obter indicadores sobre a sua satisfação com os resultados obtidos.

\section{Resultados e Discussão}

Inicialmente, foi realizado um desenvolvimento interativo e incremental da solução em ciclos quinzenais com um especialista do domínio sênior durante três meses. Então, um estudo de viabilidade foi realizado e, com base nos resultados, foram aprimoradas as versões iniciais do método semiautomatizado para extração de conhecimento de modelos de processos de negócio e geração de uma representação arquitetural de SoIS, bem como do ferramental de apoio desenvolvidos. O método proposto contempla as etapas apresentadas na Figura 1.A, que se refere ao macroprocesso do método.

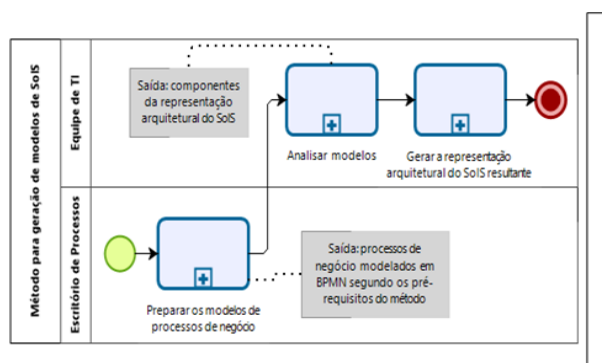

(A)

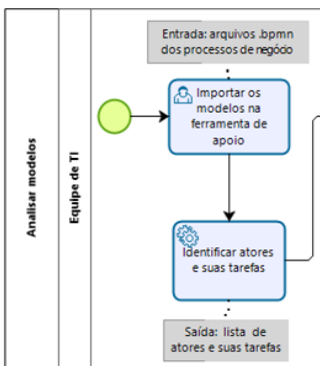

Salida: ilita de
sores e suas tarets

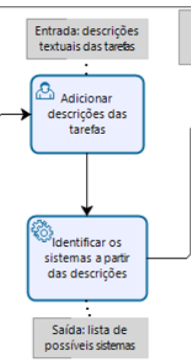

(B)

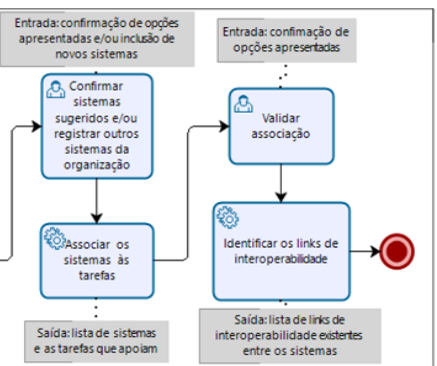

Saidat listade sistem
eastaretss que apoia

Figura 1. (A) Método Proposto e (B) Subprocesso: Analisar modelos 
Inicialmente, a equipe do Escritório de Processos, ou os responsáveis pela gestão de processos da organização, realizam a preparação dos modelos de processos de negócio, o que envolve a sua modelagem (caso esta não exista), ou a sua checagem contra requisitos definidos pelo método e necessários à sua análise. Após a preparação, a equipe de Tecnologia da Informação (TI) ou o responsável pela aplicação do método executa a análise dos modelos. Durante esta etapa, os modelos são examinados com o auxílio do ferramental de apoio e, a partir desta análise, são identificados os links de interoperabilidade entre os sistemas. A Figura 1.B apresenta o subprocesso referente à esta etapa. Por fim, com base nos resultados da análise dos modelos, deve ser gerada a representação arquitetural do SoIS (esta etapa ainda está em elaboração nesta pesquisa).

O subprocesso de análise dos modelos de processos de negócio se inicia com a sua importação pelo ferramental de apoio (Figura 2.A). Em seguida, a ferramenta extrai os dados contidos nos modelos, identificando os atores presentes nos processos e suas respectivas tarefas (Figura 2.B). Então, é solicitado ao usuário enriquecer as descrições das tarefas extraídas dos modelos. Neste ponto, são inseridos na ferramenta os detalhes sobre as tarefas (e.g., como são realizadas). A partir dessas descrições, a ferramenta efetua a identificação dos sistemas que apoiam a execução das tarefas e os apresenta ao usuário. $\mathrm{O}$ usuário efetua a confirmação das sugestões ao validar os sistemas propostos pela ferramenta, excluindo aqueles apresentados erroneamente e/ou acrescentando outros que não foram sugeridos. Além disto, o usuário deve fornecer uma descrição para cada sistema (função principal).

Com base nas descrições apresentadas, a ferramenta realiza um mapeamento automático dos sistemas às tarefas, no qual a ferramenta conecta cada tarefa ao(s) sistema(s) que apoia $(\mathrm{m})$ a sua execução e apresenta o resultado para o usuário. $\mathrm{O}$ usuário realiza em seguida a validação do mapeamento. Nesta tarefa, o usuário confirma ou corrige, se necessário, as sugestões de conexão entre tarefas e sistemas apresentadas pela ferramenta. Por fim, a ferramenta faz a identificação dos links de interoperabilidade entre os sistemas mapeados a partir das tarefas que os sistemas apoiam e do fluxo de atividades dos processos. Tais links são identificados quando tarefas consecutivas são apoiadas por diferentes sistemas, pois essa dinâmica infere que há alguma interação entre eles durante a execução do processo. Após identificados, os links de interoperabilidade são apresentados ao usuário para serem utilizados como base para a geração da representação arquitetural do SoIS resultante.

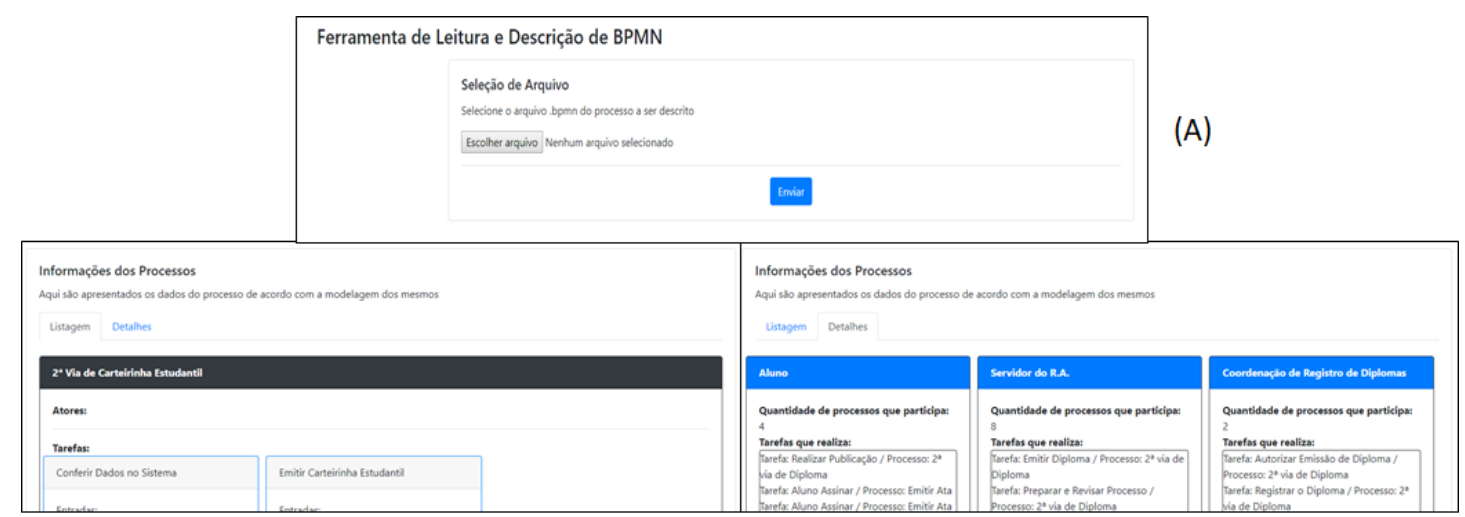

(B)

(C)

Figura 2. Interface da ferramenta 
A ferramenta de apoio ao método foi desenvolvida para automatizar as análises dos modelos. Assim, é possível inserir modelos de processo que serão analisados e terão as suas informações extraídas. A ferramenta atinge esse resultado ao fazer a leitura dos modelos extraídos com base no formato de arquivo .bpmn (padrão semelhante ao XML), em que os elementos da modelagem são representados por diferentes tags e seus atributos. A partir dessa leitura, os elementos do modelo de cada processo (como atores, tarefas etc.) são salvos em um banco de dados para, em seguida, serem cruzados e comparados. Em sua versão inicial, a interface da ferramenta se divide em duas áreas. $\mathrm{Na}$ primeira, apresentada na Figura 2.A, é feita a seleção dos arquivos a serem analisados, enquanto na segunda são expostas as informações dos processos. Neste caso, há uma listagem geral dos processos das unidades organizacionais, e de outras organizações, e seus atributos (atores, tarefas, gateways e subprocessos), presentes na Figura 2.B, e um relatório dos atores que interagem nesses processos, com suas respectivas tarefas, como ilustrado na Figura 2.C.

\section{Trabalhos Relacionados}

No trabalho de Amr et al. (2017), é proposto um modelo de interface e adaptação para interoperabilidade de múltiplos SI. O modelo disponibiliza uma camada de middleware SOA (Service-Oriented Architecture), que oculta a complexidade e heterogeneidade dos SI e é interconectada de modo a garantir a interoperabilidade. Os autores transformam os modelos de processos de negócio BPMN dos diferentes SI interconectados em BPEL (Business Process Execution Language). Em seguida, diferentes BPEL são processadas e as suas diferenças são tratadas por meio de um único banco de dados ontológico. Por fim, é estabelecida uma BPEL global (BPEL de todos os constituintes combinadas) sobre a qual será utilizada engenharia reversa para se criar um modelo de negócio BPMN global. Esta transformação funciona como uma atualização que será aplicada aos modelos de processos de cada SI constituinte para se obter sincronização de todos os SI em nível do processo de negócio. Logo, o trabalho dos autores utiliza a modelagem BPMN a fim de alcançar sincronização entre os constituintes, enquanto esta pesquisa de mestrado a utiliza para identificação de pontos de interoperação entre os sistemas.

Falcone et al. (2018) apresentam uma integração entre BPMN e padrões HLA (High Level Architecture) para a construção de SoS. No trabalho dos autores, é demonstrado como BPMN pode ser utilizado por desenvolvedores para: (i) criar uma ponte que reduz a diferença entre as atividades realizadas por um sistema federado ${ }^{1} \mathrm{e}$ sua implementação concreta; e (ii) modelar relações e fluxos de comunicação entre os sistemas federados que estão sendo executados em conjunto em uma simulação distribuída. O trabalho busca definir uma notação gráfica padronizada, baseada nas técnicas de fluxograma do BPMN, que permita aos desenvolvedores representarem de forma bem definida, em termos de modelos BPMN, tanto um sistema federado como federação HLA. Em contrapartida, nesta pesquisa de mestrado, busca-se a definição de um método que permite extrair informações dos modelos BPMN existentes.

\section{Referências}

ABPM. (2013) "Guia para o Gerenciamento de Processos de Negócio - Corpo Comum de Conhecimento". Association of Business Process Management Professionals.

\footnotetext{
${ }^{1}$ Um tipo de sistema de gerenciamento de banco de dados distribuído.
} 
Amr, M. F., Qbadou, M., Mansouri, K. e Riyami, B. (2017) "Towards a model of adaptation and interfacing based on a middleware layer SOA for interoperability of several different information systems". In: ISCV, Morocco, pp. 01-08.

Falcone, A., Garro, A., D'ambrogio, A. e Giglio, A. (2018) "Using BPMN and HLA for SoS engineering: lessons learned and future directions". In: 2018 IEEE International Systems Engineering Symposium (ISSE), Roma, pp. 01-08.

Fernandes, J., Ferreira, F., Cordeiro, F., Graciano Neto, V. e Santos, R. P. (2019) "A Conceptual Model for Systems-of-Information Systems". In: IEEE 20th Intl. Conf. on Information Reuse and Integration for Data Science (IRI), Las Vegas, pp. 364-371

Fu, X.-L., Song, M.-Q., Yu,Y.-N. e Chen, M. (2010) "Integration management view of information systems in an enterprise". In: 2nd International Conference on Information Technology and Computer Science, Kiev, pp. 317-321.

Georgantzas, N. C. e Katsamakas, E. G. (2010) "Performance effects of information systems integration: A system dynamics study in a media firm". Business Process Management Journal, v. 16, n. 5, pp. 822-846.

Graciano Neto, V. V., Cavalcante, E., El-Hachem, J. e Santos, D. S. (2017) "On the Interplay of Business Process Modeling and Missions in Systems-of-Information Systems". In: IEEE/ACM SESoS/WDES, Buenos Aires, pp. 72-73.

Hasselbring, W. (2000) "Information system integration". Communications of the ACM, v. 43, n. 6, pp. 32-38.

Krueger, I. H., Meisinger, M., Menarini, M. e Pasco, S. (2006) "Rapid Systems of Systems Integration - Combining an Architecture-Centric Approach with Enterprise Service Bus Infrastructure". In: IEEE IRI, Waikoloa, pp. 51-56.

Lana, C. A., Souza, N. M., Delamaro, M. E., Nakagawa, E. Y., Oquendo, F. e Maldonado, J. C. (2016) "Systems-of-systems development: Initiatives, trends, and challenges”. In: XLII CLEI, Valparaíso, pp. 01-12.

Majd, S., Marie-Hélène, A. e Alok, M. (2015) “An Architectural Model for System of Information Systems”. In: OTM 2015 Workshops, Rhodes, pp. 411-420.

Mendes, A., Loss, S., Cavalcante, E., Lopes, F. e Batista, T. (2018) "Mandala: an agentbased platform to support interoperability in systems-of-systems". In: 6th SESoS, Gothenburg p. 21-28.

Santos, R. P., Werner, C. e Finkelstein, A. (2018) "Ecosystems effects on softwareconsuming organizations: an experience report on two observational studies". In: 12th European Conference on Software Architecture Companion Proceedings, Madrid, pp. 1-7 (No. 23).

Teixeira, P. G., Lopes, V. H. L., Santos, R. P., Kassab, M. e Graciano Neto, V. (2019) "The status quo of systems-of-information systems". In: IEEE/ACM SESoS/WDES, Montreal, pp. 34-41.

Vaisman, A. (2013) “An Introduction to Business Process Modeling”. In: AUFAURE, M.-A.; ZIMÁNYI, E. (Eds.) Business Intelligence: Second European Summer School. Lecture Notes in Business Information Processing. Berlin, pp. 29-61. 